This item was submitted to Loughborough's Institutional Repository (https://dspace.lboro.ac.uk/) by the author and is made available under the following Creative Commons Licence conditions.

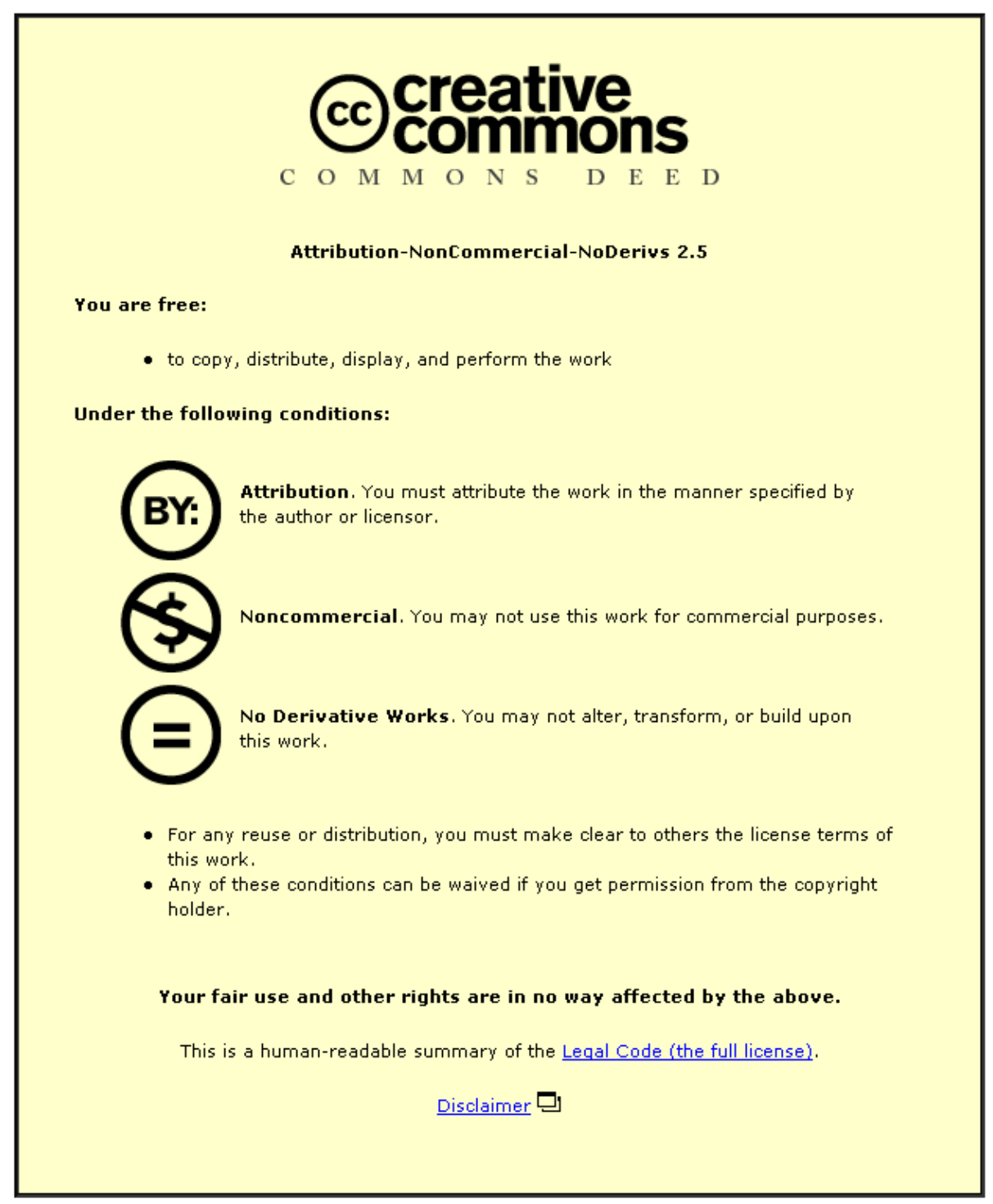

For the full text of this licence, please go to: http://creativecommons.org/licenses/by-nc-nd/2.5/ 


\section{A HIGH ACCURACY FUZZY LOGIC BASED MAP MATCHING ALGORITHM FOR ROAD TRANSPORT}

Mohammed A. Quddus

Robert B. Noland

Washington Y. Ochieng

Centre for Transport Studies

Department of Civil and Environmental Engineering

Imperial College London

LONDON SW7 2AZ

Tel: +44 2079546153

emails: m.quddus@imperial.ac.uk

r.noland@imperial.ac.uk

w.ochieng@imperial.ac.uk 


\section{Abstract:}

Recent research on map matching algorithms for land vehicle navigation has been based on either a conventional topological analysis or a probabilistic approach. The input to these algorithms normally comes from the global positioning system and digital map data. Although the performance of some of these algorithms is good in relatively sparse road networks, they are not always reliable for complex roundabouts, merging or diverging sections of motorways and complex urban road networks. In high road density areas where the average distance between roads is less than 100 metres, there may be many road patterns matching the trajectory of the vehicle reported by the positioning system at any given moment. Consequently, it may be difficult to precisely identify the road on which the vehicle is travelling. Therefore, techniques for dealing with qualitative terms such as likeliness are essential for map matching algorithms to identify a correct link. Fuzzy logic is one technique that is an effective way to deal with qualitative terms, linguistic vagueness, and human intervention. This paper develops a map matching algorithm based on fuzzy logic theory. The inputs to the proposed algorithm are from the global positioning system augmented with data from deduced reckoning sensors to provide continuous navigation. The algorithm is tested on different road networks of varying complexity. The validation of this algorithm is carried out using high precision positioning data obtained from GPS carrier phase observables. The performance of the developed map matching algorithm is evaluated against the performance of several well-accepted existing map matching algorithms. The results show that the fuzzy logic-based map matching algorithm provides a significant improvement over existing map matching algorithms both in terms of identifying correct links and estimating the vehicle position on the links.

Keywords: GPS, digital road network, map matching, optimal estimation, and fuzzy logic 


\section{INTRODUCTION}

In the last two decades, satellite navigation technology, especially the Global Positioning System (GPS), has been rapidly developed for use as a positioning technology for road transport. This includes the use of radio-navigation for route guidance, dispatching roadside assistance vehicles, accident \& emergency response, automated location tracking, scheduling of commercial vehicles, and systems for providing public transport arrival information. GPS provides 24-hour, all-weather 3D positioning and timing all over the world, with a predicted horizontal accuracy of $13 \mathrm{~m}$ (global average, signal-in-space) 95\% of the time (US DoD, 2001).

GPS , however, suffers both systematic errors or biases and random noise. A real-world field test in London showed that the GPS positioning errors sometimes could be offset from the true position by more than 50 m (100\%) (Zhao et al., 2003) while in Hong Kong it was found to be more than $80 \mathrm{~m}$ (Chen et al., 2003). A recent study to characterise the performance of GPS in a typical urban area showed $90 \%$ availability for a 4-hour trip in the Greater London area (Zhao et al., 2003). The implication of the outage involved here (i.e. 10\%) was a potential loss of navigation capability during a crucial period. In order to achieve the required accuracy and availability in some areas, GPS data can be augmented with Deduced Reckoning (DR) sensor data with the use of an Extended Kalman Filter (EKF). Zhao et al. (2003) applied an EKF to combine GPS and DR data and achieved a 100\% coverage with a 2D horizontal accuracy of $50 \mathrm{~m}(3 \sigma)$ relative to a high resolution $(1: 1,250)$ road centreline map for the same trip (Zhao et al., 2003). 
Integration of GPS and DR increases coverage but does not necessarily increase positioning accuracy (Zhao et al., 2003). Another essential element for land vehicle positioning and navigation is a digital map of the road network but these are also prone to errors (Noronha and Goodchild, 2000). For example, roads are normally represented as a single "centreline” and curvatures are represented as piecewise linear lines (for gentle curves) or as a polyline (for sharp curves). This generalisation alters the features on the ground and potentially introduces a significant bias (NRC, 2002). Therefore, Map Matching (MM) algorithms are normally used to enhance the geometric positioning accuracy of land vehicle navigation.

The most commonly used MM algorithms are based on a simple search concept. In this approach, each position fix matches to the closest 'node' or 'shape point' in the network, which is known as point-to-point matching (Bernstein and Kornhauser, 1998). These methods are easy to implement, although they are very sensitive to outliers and to the way in which the network was digitized, hence leading to errors. Another MM approach is point-to-curve matching (e.g., Bernstein and Kornhauser, 1998; White et al., 2000; Taylor et al., 2001). In this approach, the position fix from the navigation system is matched with the closest curve in the network. Although this approach gives better results than point-to-point matching, it has several shortcomings that sometimes make it inappropriate in practice, such as generating very unstable results in dense urban networks. Another geometric approach is to compare the vehicle’s trajectory against known roads. This is also known as curve-to-curve matching (Bernstein and Kornhauser, 1998; White et al., 2000). This approach is quite sensitive to outliers and depends on point-to-point matching, sometimes giving unexpected results (Greenfeld 2002). Taylor et al. (2001) proposed a novel method of map matching using GPS, height aiding from the digital terrain model (DTM) and virtual differential GPS (VDGPS) corrections, referred to as the road reduction filter ( $R R F)$ algorithm. In this approach, the 
final vehicle position is estimated without taking into account the error sources associated with the GPS position solution and the digital road network map data. The mean horizontal accuracy of the $R R F$ algorithm was found to be $14 \mathrm{~m}$. Since the study was carried out when the selective availability (S/A) was switched on, the performance of the RRF algorithm needs to be re-evaluated in the S/A free environment. Moreover, the RRF is not suitable for urban canyons as it requires at least three in-view satellites for a position solution and DTM data used in the RRF algorithm is not readily available with a desired level of accuracy needed for many transport telematics applications. Greenfeld (2002) reviews several approaches for solving the map matching problem and proposes a weighted topological algorithm. The algorithm is based on assessing the similarity between the characteristics of the street network and the positioning pattern of the user. The paper reports that the procedure computes correct matches virtually everywhere. Quddus et al. (2003) tested this algorithm for a relatively sparse road network and concluded that the algorithm identified $10 \%$ of the road segments incorrectly. Greenfeld (2002) also suggests that additional research is required to verify the performance of the algorithm.

The complexity of the MM algorithms depends on the nature of the application and the availability of data inputs. Previous research by the author and his colleagues resulted in the development of two MM algorithms. The first algorithm was based on a conventional topological analysis (Quddus et al., 2003). This map matching algorithm starts with nodal matching to identify the correct link among the links connected to the node closest to the position fix. This approach may not always be suitable for complex urban road networks with high resolution digital maps, especially when the positioning data contains outliers. The second was based on a probabilistic approach (Ochieng et al., 2004). Instead of using the links connected to the closest node, the probabilistic map matching algorithm takes all links 
as candidate links that fall within an error ellipse around a position fix. The dimensions of the error ellipse are chosen based on the error variance-covariance matrix associated with GPS/DR. The size of the error ellipse normally depends on the probability (95\% or $99 \%$ ) that the ellipse contains a true link. Although the performance of these algorithms is quite good in relatively sparse road networks, they are not suitable for complex roundabouts, merging or diverging sections of a motorway and complex urban road networks. In high road density areas where the average distance between roads is less than $100 \mathrm{~m}$, there may be many road patterns matching the trajectory of the vehicle reported by the positioning system at any given moment. Consequently, it may be difficult to precisely identify the road on which the vehicle is travelling. It is possible that these MM algorithms may suggest that the vehicle is "more likely" to be on certain roads, and "less likely" to be on other roads. Therefore, techniques for dealing with qualitative terms such as likeliness are essential in $\mathrm{MM}$ algorithms to improve the identification of correct links.

Fuzzy logic is one technique that is an effective way to deal with qualitative terms, linguistic vagueness, and human intervention (Zhao, 1997). In fuzzy logic, linguistic terms with vague concepts can be expressed mathematically by making use of fuzzy sets. A set of rules representing expert knowledge and experience is used to draw inferences through an approximate reasoning process. In MM, identification of the correct link on which the vehicle is travelling is a qualitative decision-making process involving a degree of ambiguity. Fuzzy logic based MM algorithms have been developed by a number of researchers. A complete review of other map matching algorithms can be found in Quddus (2006); a brief review of the fuzzy logic MM algorithms is given next. 
Zhao (1997) derived eight fuzzy control rules for the case in which the positioning data came from a DR sensor. The rules are a combination of those suggested by Huang et al. (1991), and Kao and Huang (1994). These methods were developed before GPS was widely available.

Syed and Cannon (2004) describe a map matching algorithm based on a fuzzy logic model using GPS/DR data. The algorithm consists of two sub-algorithms: (1) first fix mode, and (2) tracking mode. In the first sub-algorithm, a fuzzy inference system (FIS) is used to identify the correct link for the initial position fix. The characteristics of this FIS are to select a set of links which are within 50m of the GPS/DR position fix. A link is then identified based on the direction of the vehicle relative to the direction of the links and the change in heading from the gyroscope. The location of the vehicle is then determined by an orthogonal projection of the position fix onto that link. Following the identification of the first link and the location of the vehicle on it, the algorithm then goes into the second sub-algorithm. Another FIS is used to see whether the subsequent position fixes can be matched to the link identified in the first fix mode. The inputs are proximity, orientation and distance travelled by the vehicle along the link. If there is any outlier in the GPS/DR outputs or a turn is detected, then the algorithm goes back to the first fix mode.

A problem with this $\mathrm{MM}$ algorithm is that it normally takes about 30 seconds in order to complete the first fix mode. This is too lengthy for some services like en-route guidance where the vehicle can travel through a few junctions within this period. In urban areas, the algorithm may have to use the first fix mode frequently and then each time there will be a delay in identifying the first correct link. Their map matching algorithm has also not taken 
into account the error sources associated with the navigation sensors and the digital maps, and hence the determination of vehicle location is not robust.

Fu et al. (2004) developed a hybrid map matching algorithm by analysing the geometry of the road network. A fuzzy logic model is used to identify the correct link among the candidate links. Two inputs used in their FIS are: (1) the minimum distance between the position fix and the link, and (2) the difference between the vehicle direction and the link direction. The fuzzy subsets associated with the first input are very small $(<20 \mathrm{~m})$, small $(20 \mathrm{~m}-40 \mathrm{~m})$, medium $(40 \mathrm{~m}-60 \mathrm{~m})$, large $(60 \mathrm{~m}-80 \mathrm{~m})$, and very large $(>80 \mathrm{~m})$. The fuzzy subsets associated with the second input are very small $\left(<5^{0}\right)$, small $\left(15^{0}-30^{0}\right)$, medium $\left(30^{0}-45^{0}\right)$, large $\left(45^{0}-\right.$ $\left.60^{\circ}\right)$, and very large $\left(>60^{\circ}\right)$. The single output of their fuzzy inference system is the possibility of matching the position fix to a link. This simple fuzzy logic model is sensitive to measurement noise. Moreover, the vehicle heading obtained from GPS is inaccurate at low speed, as speed has not been taken into account. As the algorithm selects a link for each position fix with no reference to the historical trajectory, there is a high possibility of selecting a wrong link, especially at junctions.

The limitations of these algorithms include: (a) overlooking the connectivity among road links and the historical trajectory of the vehicle which can enhance the performance of MM algorithms (b) ignoring the error sources associated with the spatial road network and the navigation sensor, (c) failing to validate the MM algorithms to assess their performance, and (d) ignoring most of the available inputs to the fuzzy logic model.

This paper develops an improved fuzzy logic algorithm that takes account of these limitations, specifically: 
- A number of new input variables (which are readily available) are included in the proposed fuzzy logic map matching algorithm. These are: (1) the speed of the vehicle, (2) the connectivity among road links, (3) the quality of position solution e.g., HDOP, and (4) the position of a fix relative to a candidate link. These inputs are incorporated into the rules in order to improve the performance of the algorithm.

- The membership functions (MFs) are optimised using true input/output data obtained from high accuracy GPS carrier phase observations.

- Three sets of knowledge-based fuzzy rules are formulated. The first set (six rules) is for an initial map-matching process (IMP), the second set (thirteen rules) is for subsequent map-matching on a link (SMP-Link), and the third set (four additional rules) is for subsequent map-matching at a junction (SMP-Junction).

- An optimal estimation technique which takes into account error sources associated with the navigation sensors and the digital map data is used to determine the location of the vehicle on a link.

The main objective of this paper is to describe this improved MM algorithm which is based on fuzzy logic theory. The algorithm is also validated using a higher accuracy reference (truth) of the vehicle trajectory as determined by GPS carrier phase observables. The algorithm is tested on various complex urban road networks and its performance is evaluated against the performance of commonly used MM algorithms.

The paper describes the fuzzy logic-based MM algorithm in detail. This includes a brief overview of the integration of the data sources used, a brief overview of fuzzy inference systems, and then the detailed steps of the algorithm. The next section briefly describes the implementation of the algorithm using real-world data. This section also presents the 
performance of the developed MM algorithm compared with the performance of commonly used MM algorithm. Conclusions and recommendations for further avenues of study are given at the end of the paper.

\section{THE PROPOSED MAP MATCHING (MM) ALGORITHM}

The capability to identify the physical location of a vehicle on a link is a key requirement in any transport telematics applications. In order to achieve the RNP, system and sensor complementarity, such as in the case of the integration of GPS, DR, and digital map data (Figure 1) that could be used to enhance geometric positioning capability. This is achieved by a MM algorithm.

\section{<Figure 1 should be placed about here $>$}

The integration of GPS and DR is achieved via an Extended Kalman Filter (EKF) algorithm as described in Zhao et al. (2003). The EKF algorithm takes inputs from the GPS and DR (the gyro-rate reading $(\Delta \theta)$, the odometer reading $(\Delta d)$ and the errors associated with them $\left(\varepsilon_{\theta}\right.$ and $\left.\varepsilon_{\mathrm{d}}\right)$. The outputs of GPS/DR are Easting $(E)$, Northing $(N)$, vehicle speed $(v)$, heading $(\theta)$, and the error variances associated with them (i.e., $\hat{\sigma}_{e}, \hat{\sigma}_{n}, \hat{\sigma}_{v}, \hat{\sigma}_{h}$ respectively). The MM algorithm takes inputs from the GPS/DR and the digital map data (e.g., topology, links, nodes, and the error variance of map data, $\left.\sigma_{\text {map }}\right)$. The outputs of the MM process are the

correct link ID, the position solution (Easting, $\hat{E}_{\text {link }}$ and Northing, $\hat{N}_{\text {link }}$ ) on that link and the uncertainty associated with the position solution $\left(\hat{\sigma}_{m m}\right)$. The MM process not only enables 
the physical location of the vehicle to be identified but also improves the positioning capability if a good digital map is available.

The MM algorithm developed in this paper has two stages: (1) the identification of the correct link and (2) the determination of the vehicle location on the selected link. These are explained below, after a brief introduction to fuzzy inference systems (FIS).

\section{Fuzzy Inference Systems (FIS)}

Fuzzy logic is a superset of conventional (Boolean) logic that has been extended to handle the concept of partial truth. It was introduced in the 1960's by Zadeh (1965). A comprehensive review of fuzzy logic theory can be found in Zadeh (1965, 1973, 1989), Mamdani and Assilian (1975), and Sugeno (1985). However, a very brief overview is presented here.

Figure 2 shows a fuzzy inference system (FIS) which is the process of formulating the mapping from a given input to an output using fuzzy logic. The mapping provides a basis from which a decision can be made.

$<$ Figure 2 should be placed about here $>$

There are two main types of FIS: (1) Mamdani-type (Mamdani and Assillian, 1975) and (2) Sugeno-type (Sugeno, 1985). A Sugeno-type FIS is used in this study and is briefly described.

A generic rule for Sugeno's FIS is as follows: 


$$
\text { If } x \text { is } A \text { and } y \text { is B then } z=f(x, y)
$$

where $x, y$ are the input variables, and $z$ is the output variable which is either a first order polymonial (known as a ' 1 st - order Sugeno fuzzy model') or a constant (known as a 'zeroorder Sugeno fuzzy model'). A and B are the fuzzy subsets of $x$ and $y$ respectively.

\section{Identification of the Correct Link}

The most complex element of any MM algorithm is to identify the actual link among the candidate links (Greenfeld, 2002; Quddus et al. 2003, and Ochieng et al., 2004). Therefore, a novel approach consisting of two distinct processes was developed for the identification of the correct link. The processes are: (1) the initial map-matching process (IMP) and (2) the subsequent map-matching process (SMP). Both of these processes are described below.

\section{Initial map-matching process (IMP)}

The selection of an initial link for the initial position fix is known as an initial map-matching process (IMP). If an initial matching is incorrect then the subsequent matching will also be incorrect. Therefore, a more sophisticated method is employed for the IMP. The IMP approach used in this paper is a function of the GPS receiver's time-to-first-fix (TTFF), the search space based on the error ellipse derived from the error variances, the perpendicular distance from a position fix to the link, the bearing of the link, and the direction of the vehicle. A few first good position fixes on a link are used to identify the first link. This increases the level of confidence that the IMP is robust.

The IMP begins just after the initialization of the GPS receiver (which may take a minute or two after switching it on depending on the surrounding environment). The basic characteristic of the IMP is the use of an elliptical or rectangular confidence region around a position fix 
based on error models associated with GPS/DR. Road links that are within the confidence region are taken as the candidate links. If the confidence region does not contain any link, then it is assumed that the vehicle is off the known road links. In such a situation, the derived GPS/DR position is used as the final location of the vehicle. In a situation where the confidence region contains only one segment, then the final selection process is very straightforward. In the case of more than one link, a fuzzy inference system (FIS) can be used to identify the correct link among the candidate links.

The most important variables available during IMP are the heading error (HE), which is defined as the absolute difference between the direction of the vehicle and the direction of the link, and the perpendicular distance (PD) from the position fix to the link. These two variables could be used as potential inputs for the FIS. However, the quality of the direction of vehicle data largely depends on the speed of the vehicle (Quddus et al., 2004, and Taylor et al., 1999). Therefore, the speed of the vehicle could be used as an additional input to the FIS. The satellite geometric contribution to the positioning error as determined by the horizontal dilution of precision (HDOP) could also be used as a quality indicator of the position fix.

Therefore, the state input variables of this FIS are: (1) the speed of the vehicle, $v(\mathrm{~m} / \mathrm{sec}),(2)$ the heading error, HE (degree), (3) the perpendicular distance, PD (m), and (4) the HDOP. The speed of the vehicle can be obtained from GPS/DR and the fuzzy subsets associated with this variable are zero, low and high. The direction of the link is obtained from the spatial road network data and the direction of the vehicle is obtained from the GPS/DR. The fuzzy subsets related to the heading error are small and large. The PD is calculated as the minimum Euclidian distance between the position fix and the link. The fuzzy subsets associated with 
this variable are low and high. The HDOP could also be obtained from the GPS/DR and the fuzzy subsets are good and bad. The four system state input variables are fuzzyfied as shown in Figure 3. Z-shaped and S-shaped MFs are chosen in the fuzzyfication process. The single output of this FIS is the likelihood of matching the position fix to a link (denoted as L1). A zero-order Sugeno fuzzy model is considered which takes three constants for the output, $L 1$ e.g., low $(\mathrm{Z1})=10$, average $(\mathrm{Z} 2)=50$ and high $(\mathrm{Z3})=100$.

\section{<Figure 3 should be placed about here $>$}

The next step is to formulate the fuzzy rules which are related to the number of system state variables. Experience and engineering knowledge are used to formulate the fuzzy rules. Special attention is given to the overlap between the fuzzy subsets of a particular input. This can reduce the number of fuzzy rules as every input generates some response. The following six rules are found to provide the best performance of the IMP. The weight of each rule is shown within the brackets at the end of each rule. Quddus et al. (2003) and Greenfeld (2002) suggest that the heading error should be given more weight than the PD. Therefore, a higher weight is given to the rules associated with the heading error.

- If ( $v$ is high) and ( HE is small) then (L1 is average) (3)

- If ( $v$ is high) and (HE is large) then (L1 is low) (1)

- If (HDOP is good) and (PD is short) then (L1 is average) (1)

- If (HDOP is good) and (PD is long) then (L1 is low) (1)

- If ( HE is small) and (PD is short) then ( L1 is high) (1)

- If ( HE is large) and ( PD is long) then (L1 is low) (1) 
The min (minimum) method is used to derive the "degree of applicability" $\left(\omega_{i}\right)$ of each fuzzy rule. The weighted average method is used to obtain a crisp output (see Quddus (2006) for details). This crisp output is the likelihood associated with a link. The FIS is applied to all links within the confidence region. The link which gives the highest likelihood is taken as the correct link among the candidate links.

The above FIS is used to identify a link on which the vehicle is travelling. Since only a few inputs are available during IMP, the link identified by the FIS for the first position fix may not be the actual link. Therefore, the IMP is performed for a few first good position fixes. If the FIS identifies the same link for those position fixes, then the link is chosen as a first correct link as illustrated in Figure 4.

<Figure 4 should be placed about here $>$

Assume that the first good position fix from the GPS/DR is denoted by the point A (Figure 4). The FIS identifies link 1 as a correct link for this position fix. The FIS then identifies link 1 as the correct link for the subsequent position fixes B, C, and D. Therefore, it can be said that the correct link for the position fix, D, is link 1.

\section{The subsequent map-matching process (SMP)}

After successfully implementing the IMP, the subsequent map-matching process (SMP) is used. The basic function of SMP is to match the following position fixes. Two types of SMPs are proposed: (1) SMP along a link (SMP-1) and (2) SMP at a junction (SMP-2). The purpose 
of SMP-1 is to match the subsequent position fixes with the link identified by IMP unless the vehicle is either about to cross or has already crossed a junction. The purpose of SMP-2 is to identify a new link among the candidate links at a junction for the last non-matched position fix. After identifying a new link by the SMP-2, the SMP-1 restarts to match the subsequent position fixes to the new link. Both of these processes are explained below.

SMP along a link (SMP-1)

The SMP-1 starts to match the following position fixes on the previously selected link, which is identified using IMP (or SMP-2). In SMP-1, a Sugeno FIS is used to see whether the following position fixes could be matched on the previously selected link. The SMP-1 is a function of the direction of the vehicle, the gyro-rate reading, the distance along the link from the last matched position fix to the downstream junction, and the speed of the vehicle.

\section{<Figure 5 should be placed about here $>$}

Figure 5 shows the state variables of the vehicle travelling on a link. Point A represents the last matched vehicle position on link 1 which was identified by IMP (or SMP-2). Therefore, the task of SMP-1 is to select the correct link for the subsequent position fix, B. The term $d 1$ refers to the distance from the last matched vehicle position to the downstream junction and $d 2$ refers to the distance travelled by the vehicle within the last second which can be calculated from vehicle speed at B. The difference between these two distances, $\Delta d=(d 1$ $d 2$ ), can be used to see whether the vehicle crosses a junction. For example, if $\Delta d$ is negative, then it is more likely that the vehicle has already crossed the junction. The $\alpha$ and $\beta$ define the location of the position fix, B, relative to link 1 . If both of these angles are less than $90^{\circ}$, then it is more likely that the vehicle has not crossed the junction. The angles $\theta$ and $\theta^{\prime}$ indicate 
the directions of the vehicle at B and A respectively. The absolute difference between these two angles i.e., $a b s\left(\theta-\theta^{\prime}\right)$ gives a heading increment at $\mathrm{B}$ for the last epoch. The lower the heading increment the higher is the possibility that the vehicle is on link 1 . If the heading increment is close to zero, then it is more likely that there is no left or right turn. In addition, the gyro-rate reading at $\mathrm{B}$, which is the rate of change of heading for the last epoch, can also be used to see whether there is a left or right turn.

Therefore, the fuzzy variables of this FIS are: (1) the speed of the vehicle, $v(\mathrm{~m} / \mathrm{sec})(2)$ the heading increment (HI) (degree) (3) the gyro-rate reading, $\Delta \theta$ (deg/sec) (4) the $\Delta d(\mathrm{~m})(5)$ the value of $\alpha$ (degree) (6) the value of $\beta$ (degree), and (7) the HDOP. The single output of this FIS is denoted as $L 2$ and is the possibility of matching the current point (in this example, B) on the previously identified link (in this example, link 1) denoted by $L 2$. The fuzzyfication of the state input variables is shown in Figure 6. Z-shaped, S-shaped and gauss MFs (for the $180^{\circ}$ heading increment) are used in the fuzzyfication process. A zero-order Sugeno fuzzy model is considered which takes three constants for the output $L 2$ e.g., $\operatorname{low}(Z 1)=10$, $\operatorname{average}(\mathrm{Z} 2)=50$ and $\operatorname{high}(\mathrm{Z} 3)=100$.

\section{<Figure 6 should be placed about here $>$}

A sensitivity test was carried out to determine the optimal number of rules that provide the best performance for identification of the correct link at a junction. This is achieved using a true input/output dataset. Both the sensitivity analysis procedure and the rules are transferable. A full discussion of this can be found in Quddus (2006). The following thirteen rules were selected. 
- If ( $\Delta \theta$ is small) and ( $\alpha$ is below $90^{\circ}$ ) and ( $\beta$ is below $\left.90^{\circ}\right)$ then ( $L 2$ is high) (1)

- If ( $\Delta \theta$ is small) and ( $\Delta d$ is positive) and ( $\alpha$ is above $90^{\circ}$ ) then ( $L 2$ is low) (1)

- If ( $\Delta \theta$ is small) and ( $\Delta$ d is positive) and ( $\beta$ is above $90^{\circ}$ ) then ( $L 2$ is low) (1)

- If ( $\mathrm{HI}$ is small) and ( $\alpha$ is below $90^{\circ}$ ) and ( $\beta$ is below $\left.90^{\circ}\right)$ then (L2 is high) (1)

- If (HI is small) and ( $\Delta d$ is positive) and ( $\alpha$ is above $90^{\circ}$ ) then (L2 is low) (1)

- If (HI is small) and ( $\Delta$ d is positive) and ( $\beta$ is above $\left.90^{\circ}\right)$ then ( $L 2$ is low) (1)

- If ( $\Delta \theta$ is high) and ( $\alpha$ is below $\left.90^{\circ}\right)$ and $\left(\beta\right.$ is below $\left.90^{\circ}\right)$ then (L2 is low) (1)

- If (HI is large) and ( $\alpha$ is below $\left.90^{\circ}\right)$ and $\left(\beta\right.$ is below $\left.90^{\circ}\right)$ then ( $(\mathrm{L} 2$ is low) (1)

- If (HDOP is good) and ( $v$ is zero) then (L2 is high) (1)

- If (HDOP is good) and ( $\triangle d$ is negative) then (L2 is average) (1)

- If (HDOP is good) and ( $\triangle d$ is positive) then (L2 is low) (1)

- If ( $v$ is high) and (HI is small) then (L2 is average) (1)

- If (HDOP is good) and ( $v$ is high) and (HI is $\left.180^{\circ}\right)$ and ( $\Delta \theta$ is high) then (L2 is high) (1)

The last rule is included to determine whether a vehicle makes a U-turn at a junction. A threshold output value can be used to determine whether the position fix should match with the previously selected link. The threshold value can empirically be derived by applying the FIS to a given (true) input/output dataset. 
SMP at a junction (SMP-2)

The SMP-2 begins when the vehicle is either about to cross or has just crossed a junction. A new link is determined among the candidate links using the same FIS described in IMP. However, two more input variables are available at this moment. These are the link connectivity and the distance error as shown in Figure 7.

\section{<Figure 7 should be placed about here $>$}

Assume that the vehicle is travelling on link 1. The last map-matched position on that link is

S. The task of SMP-2 is to select a new link for the position fix, C, as the vehicle has already crossed the junction. The candidate links for this position fix are 2, 3 and 6. Since the location of the vehicle at the previous epoch is on link 1 , the link connectivity helps to identify the correct link. For example, there is no direct connection between links 1 and 6. Therefore, it is unlikely that the vehicle is on link 6 for the position fix C. The term $d$ refers to the distance travelled by the vehicle within the last second which can be calculated by the speed of the vehicle at C. The $d_{2}, d_{3}$, and $d_{6}$ represent the shortest paths travelled by the vehicle if the vehicle is on links 2, 3 and 6 respectively. The difference between $d$ and $d_{2}$ (or $d_{3}$ or $d_{6}$ ) is the distance error associated with each of the link as shown in Figure 7. The distance error is an input to the FIS. For example, the link which gives the lowest distance error is a strong candidate for the correct link. The two additional state input variables are fuzzyfied as shown in Figure 8. The output variable of this FIS is the likelihood of matching to a link (L3). A zero-order Sugeno fuzzy model is considered which takes three constants for the output L3, e.g., low $(Z 1)=10$, average $(Z 2)=50$ and high $(Z 3)=100$. 


\section{$<$ Figure 8 should be placed about here $>$}

Ten rules are used for this FIS. The first six rules are the same as presented in the FIS of IMP. The rest of the rules are given below.

- If (The connectivity with the previous link is low) then (The L3 is low) (1)

- If (The connectivity with the previous link is high) then (The L3 is high) (1)

- If (The distance error is low) then (The L3 is high) (1)

- If (The distance error is high) then (The L3 is low) (1)

The FIS is applied to all links within the error region and the link which gives the highest likelihood value is taken as the correct link.

\section{Determination of the Vehicle Location on the Selected Link}

Assuming that the correct link has been identified as per the IMP and/or SMP, the physical location of the vehicle on the link can be determined in two ways with the available data. One method is to use map data (i.e., link heading) and vehicle speed from the positioning sensors. If an initial position for the vehicle is known then the vehicle position (easting, $e_{\text {map }}$, northing, $n_{\text {map }}$ ) can be derived epoch-by-epoch from the link heading and speed information. The other method is to adopt the perpendicular projection of the GPS or the GPS/DR fix on to the link that results in the easting $\left(e_{g p s}\right)$ and northing $\left(n_{g p s}\right)$ coordinates. Since both methods are associated with errors, an optimal estimation procedure (combining the two methods) is used 
to determine the final location of the vehicle on the road segment. The optimal easting $(\hat{e})$ and northing ( $\hat{n}$ ) for a particular epoch are expressed as

$$
\begin{aligned}
& \hat{e}=\left(\frac{\sigma_{g p s, e}^{2}}{\sigma_{\text {map }}^{2}+\sigma_{g p s, e}^{2}}\right) e_{\text {map }}+\left(\frac{\sigma_{\text {map }}^{2}}{\sigma_{\text {map }}^{2}+\sigma_{g p s, e}^{2}}\right) e_{g p s} \\
& \hat{n}=\left(\frac{\sigma_{g p s, n}^{2}}{\sigma_{\text {map }}^{2}+\sigma_{g p s, n}^{2}}\right) n_{\text {map }}+\left(\frac{\sigma_{\text {map }}^{2}}{\sigma_{\text {map }}^{2}+\sigma_{g p s, n}^{2}}\right) n_{g p s}
\end{aligned}
$$

where $\sigma_{\text {map }}^{2}$ is the error covariance associated with map data, $\sigma_{g p s, e}^{2}$ and $\sigma_{g p s, n}^{2}$ are the easting and northing components of the error covariance associated with the navigation sensor. The error variance associated with $\hat{e}$ can now be expressed as

$$
\frac{1}{\sigma_{m m, e}^{2}}=\frac{1}{\sigma_{\text {map }}^{2}}+\frac{1}{\sigma_{g p s, e}^{2}}
$$

where $\sigma_{m m, e}^{2}$ is the error variance associated with optimal estimation of $\hat{e}$. Note from equation (3) that $\sigma_{m m, e}^{2}$ is less than either $\sigma_{\text {map }}^{2}$ or $\sigma_{g p s, e}^{2}$. That is, the uncertainty in the estimation of the vehicle position using optimal estimation is decreased by combining two measurement methods. Similarly, the error variance associated with the optimal estimation of $\hat{n}$ can also be derived from equation (3). 


\section{RESULTS}

A comprehensive field test was carried out to collect positioning data from various road environments including motorway merging/diverging scenarios, complex roundabouts, and complex urban roads. This is necessary because the performance of MM algorithms largely depends on road network characteristics. The test route was chosen carefully to have a good mix of important spatial urban characteristics including open spaces, urban canyons, tall buildings, tunnels, bridges, and potential sources of electromagnetic interference. The duration of data collection was about 4 hrs. In order to validate the proposed positioning algorithms, the carrier phase observables from GPS are used. For this test, the route was selected carefully to have good satellite visibility as GPS carrier phase observables require observations from a large number of GPS satellites (Ochieng et al., 2004). The duration of GPS carrier phase data collection was about 2 hrs.

A vehicle was equipped with a navigation platform consisting of a 12-channel single frequency high sensitivity GPS receiver, a low-cost rate gyroscope and the interfaces required to connect to the vehicle speed sensor (odometer) and back-up indicator. In order to obtain the reference (truth) trajectory by GPS carrier phase observables, the vehicle was also equipped with a 24-channel dual-frequency geodetic receiver. High accuracy local measurement of 3-D offsets between the two antennae was undertaken in order that the position information was referenced to a single point. 
The positioning data (easting and northing), speed, heading and associated error variances were collected at one-second intervals directly from the integrated navigation system (GPS/DR sensors) employing an Extended Kalman Filter (EKF) algorithm

The fuzzy logic-based MM algorithm was tested for various scenarios with different network characteristics and with different traffic manoeuvres. Only a complex roundabout with a motorway diverging (Figure 9) and a complex urban road network (Figure 10) are shown here as an example. Each of the blue round dots in the Figures (9 and 10) represents the vehicle position before MM. The arrow symbols in the figures show the path followed by the vehicle on the network. Each of the triangular symbols on the road segments represents the vehicle position after MM.

\section{$<$ Figure 9 should be placed about here $>$}

A threshold value of 60 for the likelihood (L2) (the output of FIS in SMP-1) was found to be adequate to identify whether the vehicle position should match with the previously selected road segment. Assuming the duration of a typical trip length is about 30 minutes, this threshold value was empirically determined from a true input/output dataset of 1812 epochs. The MFs of all input variables was also trained and modified with the same input/output dataset using the fuzzy logic toolbox of Matlab (MathWorks, 2000). The CPU processing time required to train the MFs using such a dataset was less than 6 sec. The position of the vehicle on a selected road segment was estimated using two positioning methods (estimation using map data with the vehicle speed information from the positioning unit and the other is from the orthogonal projection of the GPS/DR fix on the road segment as shown in equations 
(1) and (2). The reliability test was performed after matching each position fix to the centreline of the road network map.

\section{<Figure 10 should be placed about here $>$}

The MM algorithm was validated using the validation strategy explained in Quddus et al. (2004). The GPS carrier phase measurements were used as the reference (truth) of the vehicle positions. A set of correct links on which the vehicle was travelling was identified based on the true vehicle trajectory. Another set of links was identified for the corresponding epochs from the developed MM algorithm. The results showed that the fuzzy logic-based MM algorithm identified $99.2 \%$ of the links correctly (4570 correct links out of 4605 total links).

In terms of physical location of the vehicle, different categories of horizontal positioning errors were derived. The errors associated with the positions from the GPS augmented with DR were within $34 \mathrm{~m}$ relative to the truth positions. The horizontal accuracy was $10.3 \mathrm{~m}(2 \sigma)$ and the standard deviation was $6.2 \mathrm{~m}$. The next step was to compute the horizontal errors associated with the positions estimated from the developed MM algorithm. It was found that all MM positions on the road centreline were within 11m (maximum error) of the truth positions of the vehicle. The horizontal positioning accuracy was $5.5 \mathrm{~m}$ (95\%) and the standard deviation of the errors was $2.3 \mathrm{~m}$. The positioning accuracy of the along-track component of the error was $4.2 \mathrm{~m}$ (95\%) and the cross-track component of the error was 3.2m (95\%). 
The performance of the map matching algorithm developed in this paper was also evaluated against the performance of other existing map matching algorithms. The most common map matching algorithms in the literature are point-to-point matching (Bernstein and Kornhauser, 1998), point-to-curve matching (White et al., 2000), improved point-to-curve matching (Srinivasan et al. 2003), weighted topological analysis (Greenfeld, 2002), advanced weighted topological analysis (Quddus et al., 2003), Dempster-Shafer's (D-S) theory of evidence (Yang et al., 2003), and other fuzzy logic based models (Fu et al., 2004; Syed and Cannon, 2004).

\section{<Table 1 should be placed about here>}

The data collected from a sub-urban area are used to examine the performance of these algorithms in terms of the identification of correct links and the determination of 2-D horizontal accuracy together with the along-track and the cross-track errors. The method discussed above is used to quantify the performance of the map matching algorithms. The navigation data used to evaluate the performance of the algorithms is from GPS/DR and the digital map data is from the map of scale 1:2500. The results are shown in Table 1 . The second column represents the percentage of correctly matched links. The third, fourth and

fifth columns show horizontal accuracy (95\%), along-track errors (95\%, m) and cross-track errors $(95 \%, \mathrm{~m})$ respectively. As can be seen, the MM algorithm developed in this research outperforms the other algorithms on all these criteria, including those algorithms were also based on fuzzy logic methods. This improvement is primarily due to the use of additional information, such as speed, error sources associated with navigation sensors and map data, and more sophisticated fuzzy rules. 


\section{CONCLUSIONS}

A fuzzy logic-based MM algorithm was developed for land vehicle navigation. In this algorithm, the factors considered to build various knowledge-based IF-THEN rules were the speed, heading and historical trajectory of the vehicle, the connectivity and the orientation of the links and the satellite geometric contribution to the positioning error (HDOP). A Sugenotype fuzzy inference system was used to develop the algorithm and the membership functions were trained and modified using a given input/output dataset obtained from GPS carrier phase observations. The inputs to the MM algorithm were taken from an integrated navigation system (GPS/DR) in order to attain vehicle location data continuously. The MM algorithm was tested in different road networks with varying complexity. A digital road centreline map of scale 1:2,500 was used to match the vehicle position on roads. The accuracy of the proposed MM algorithm was validated against high accuracy GPS carrier phase observables on a network of $204 \mathrm{sq} \mathrm{km}$ in London. It was found that the algorithm identified $99.2 \%$ of the road segments correctly with a horizontal accuracy of $5.5 \mathrm{~m}(2 \sigma)$, a significant improvement over previous algorithms which were also tested.

The performance of the developed fuzzy logic based map matching algorithm in terms of positioning accuracy was not evaluated under urban conditions. This is because of the unavailability of high accuracy GPS carrier-phase observations in central London and most urban areas. Future research could utilize a high-grade Inertial Navigation System (INS) integrated with GPS that would have the potential to derive the true vehicle trajectory in urban areas. The threshold value for L2 was derived empirically from a series of field observations. Therefore, the value could vary in different operational environments. Future research will also consider the integrity of map matching. This will include the specification 
of a metric for measuring the quality (and level of confidence of map matching) and the detection of anomalies (in raw and positional data).

\section{REFERENCES}

Bernstein, D., Kornhauser, A. (1998). Map matching for personal navigation assistants. In proceedings of the 77th annual meeting of the Transportation Research Board, 11-15 January, Washington D.C.

Chen, W., YU, M., LI, Z.-L., Chen, Y.-Q. (2003). Integrated vehicle navigation system for urban applications. GNSS 2003, Graz, April , CD-ROM, 15 pp.

Fu, M., Li, Jie, Wang, M., (2004), A hybrid map matching algorithm based on fuzzy comprehensive Judgment, IEEE Proceedings on Intelligent Transportation Systems, 613-617.

Greenfeld, J.S. (2002). Matching GPS observations to locations on a digital map. In proceedings of the 81st Annual Meeting of the Transportation Research Board, January, Washington D.C.

Huang, L.-J., Kao, W.W., Oshizawa, H., Tomizuka, M., (1991). A fuzzy logic based map matching algorithm for automotive navigation systems, IEEE Roundtable Discussion on Fuzzy and Neural Systems, and Vehicle Applications, Paper No., 16.

Kao, W.W., Huang, L.-J., (1994). System and method for locating a travelling vehicle, United States Patent No., 5283575. 
Mamdani, E.H. and S. Assilian. (1975). An experiment in linguistic synthesis with a fuzzy logic controller. International Journal of Man-Machine Studies, 7 (1), 1-13.

MathWorks (2000). Fuzzy logic toolbox user’s guide. The Mathworks inc., Natick, MA.

Noronha, V., Goodchild, M.F. (2000), Map accuracy and location expression in transportation reality and prospects. Transportation Research C, 8, 53-69.

NRC, National Research Council (2002). Collecting, processing and integrating GPS data with GIS. NCHRP Synthesis 301. National Academy Press; Washington D.C.

Ochieng, W.Y., Quddus, M.A., Noland, R.B. (2004). Integrated positioning algorithms for transport telematics applications. In proceedings of the Institute of Navigation (ION) annual conference, 20-24 September, California, USA.

Quddus, M. A. (2006). High integrity map matching algorithms for advanced transport telematics applications, PhD thesis, Centre for Transport Studies, Imperial College London.

Quddus, M.A., Ochieng, W.Y., Zhao, L., Noland, R.B. (2003). A general map matching algorithm for transport telematics applications. GPS Solutions, 7(3), 157-167.

Quddus, M.A., Noland, R.B., Ochieng, W.Y. (2004). Validation of map matching algorithm using high precision positioning with GPS. Journal of Navigation 58, 257-271.

Sugeno, M. (1985). Industrial applications of fuzzy control, Elsevier Science Pub. Co. 
Syed, S., Cannon, M.E. (2004). Fuzzy logic-based map matching algorithm for vehicle navigation system in urban canyons. In proceedings of the Institute of Navigation (ION) national technical meeting, 26-28 January, California, USA.

Taylor, G., Blewitt, G., Steup, D., Corbett, S., Car, A. (2001). Road reduction filtering for GPS-GIS Navigation, Transactions in GIS, ISSN 1361-1682, 5(3), 193-207.

Transport for London (TfL). (2004). London Busses-Countdown.

http://www.tfl.gov.uk/buses/ini_countdown.shtml, accessed July 2004.

US DoD. (2001). Global positioning system standard positioning service performance standard. Assistant secretary of defense for command, control, communications, and intelligence.

White, C.E., Bernstein, D., Kornhauser, A.L. (2000). Some map matching algorithms for personal navigation assistants. Transportation Research Part C 8, 91-108.

Yang, D., Cai, B., Yuan, Y. (2003). An improved map-matching algorithm used in vehicle navigation system, IEEE Proceedings on Intelligent Transportation Systems, 2, 1246-1250.

Zadeh, L.A. (1965). Fuzzy sets, Information and Control, 8, 338-353.

Zadeh, L.A. (1973). Outline of a new approach to the analysis of complex systems and decision processes. IEEE Transactions on Systems, Man, and Cybernetics, 3(1), 28-44. 
Zadeh, L.A. (1989). Knowledge representation in fuzzy logic. IEEE Transactions on Knowledge and Data Engineering, 1, 89-100.

Zhao, Y. (1997). Vehicle location and navigation system. Artech House, Inc., MA.

Zhao, L., Ochieng, W.Y., Quddus, M.A, Noland, R.B. (2003). An extended Kalman filter algorithm for integrating GPS and low-cost dead reckoning system data for vehicle performance and emissions monitoring. The Journal of Navigation, 56, 257-275. 


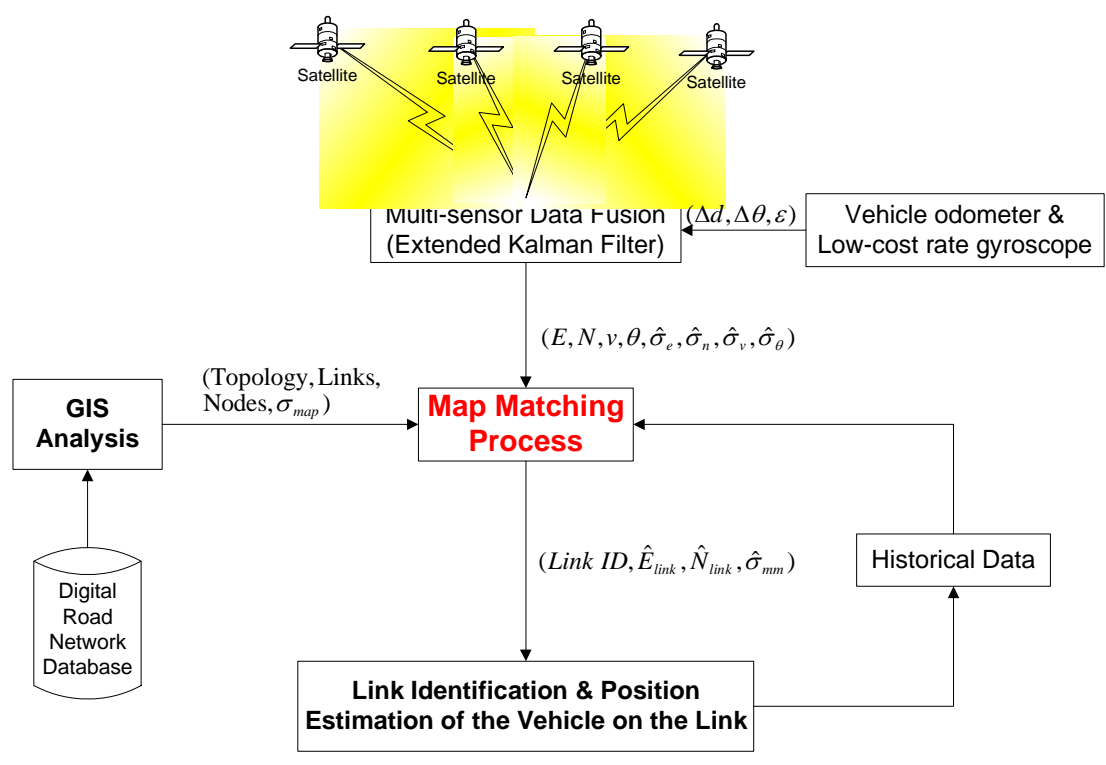

Figure 1: A schematic diagram of the GPS/DR/MM Process 


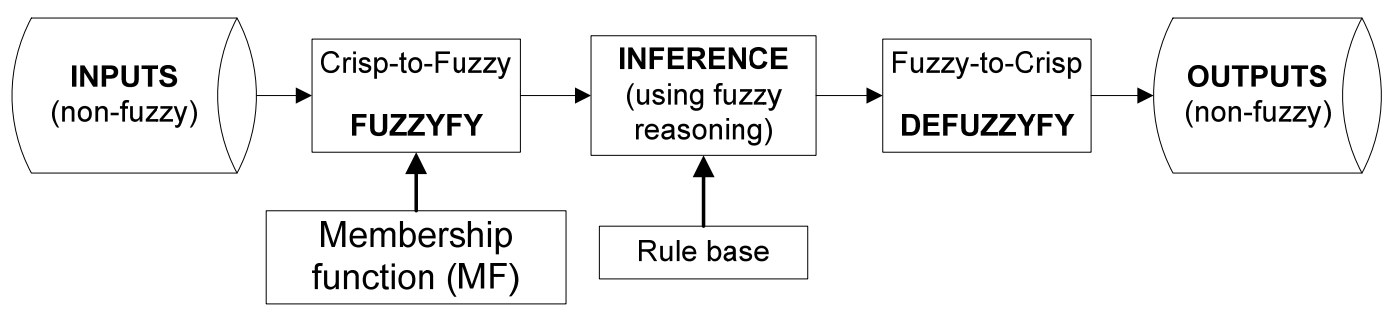

Figure 2: Fuzzy inference system (FIS) 


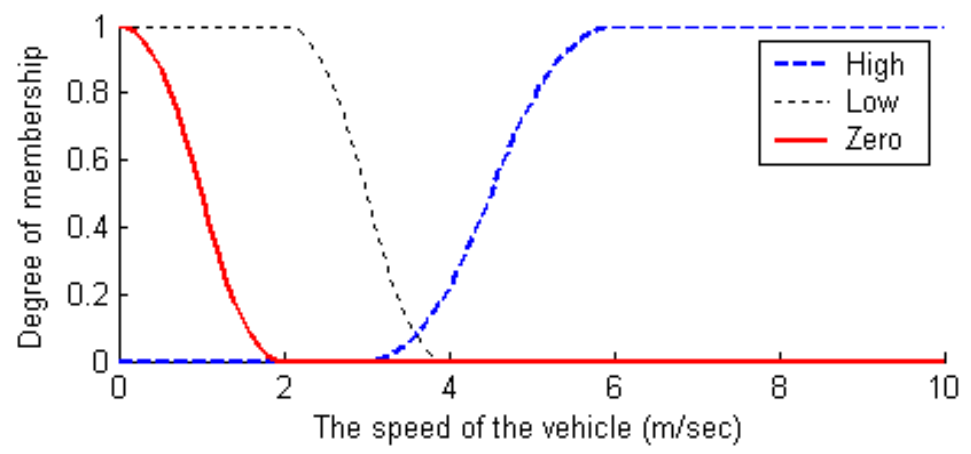

(a) 


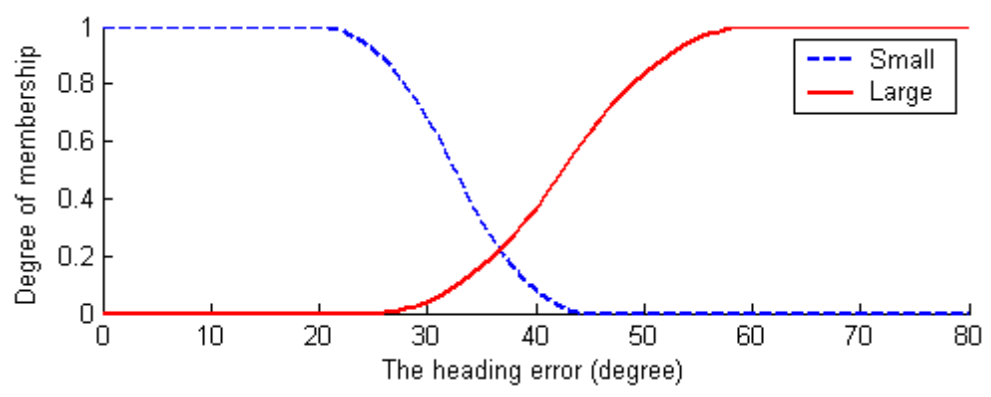

(b)

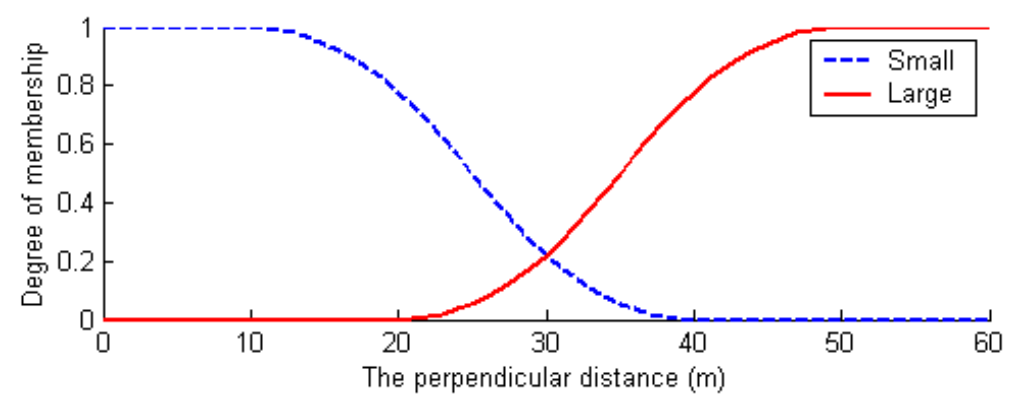

(c)

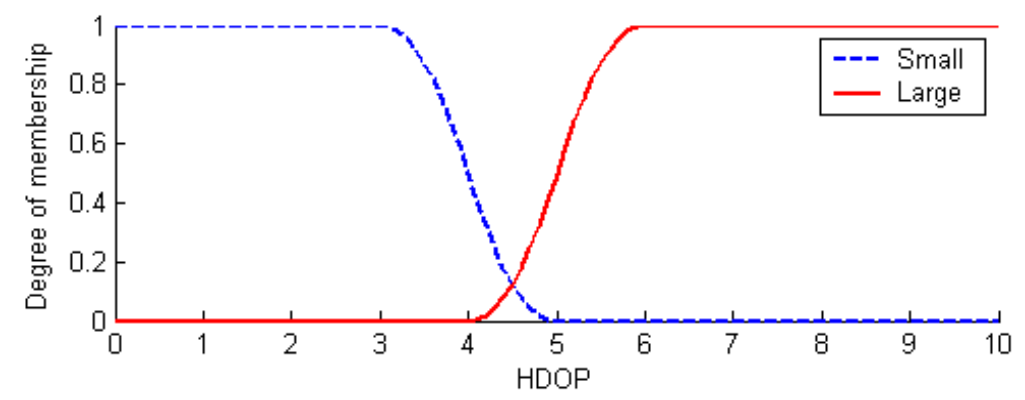

(d)

Figure 3: The fuzzification of the speed of the vehicle (a), the heading error (b), the perpendicular distance (c), and HDOP (d) 


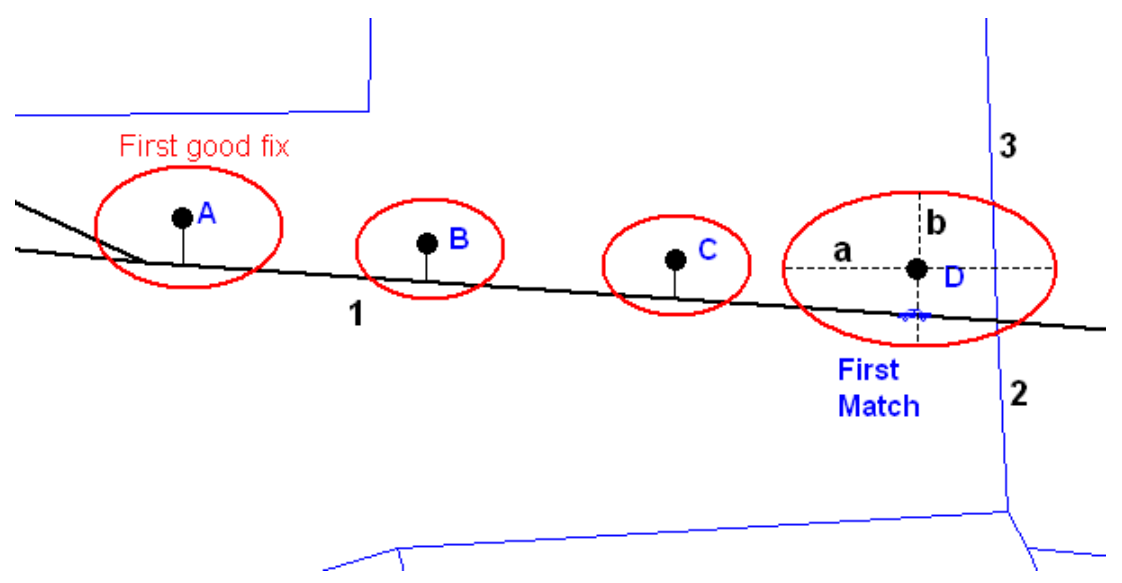

Figure 4: Example of initial map-matching process (IMP) 


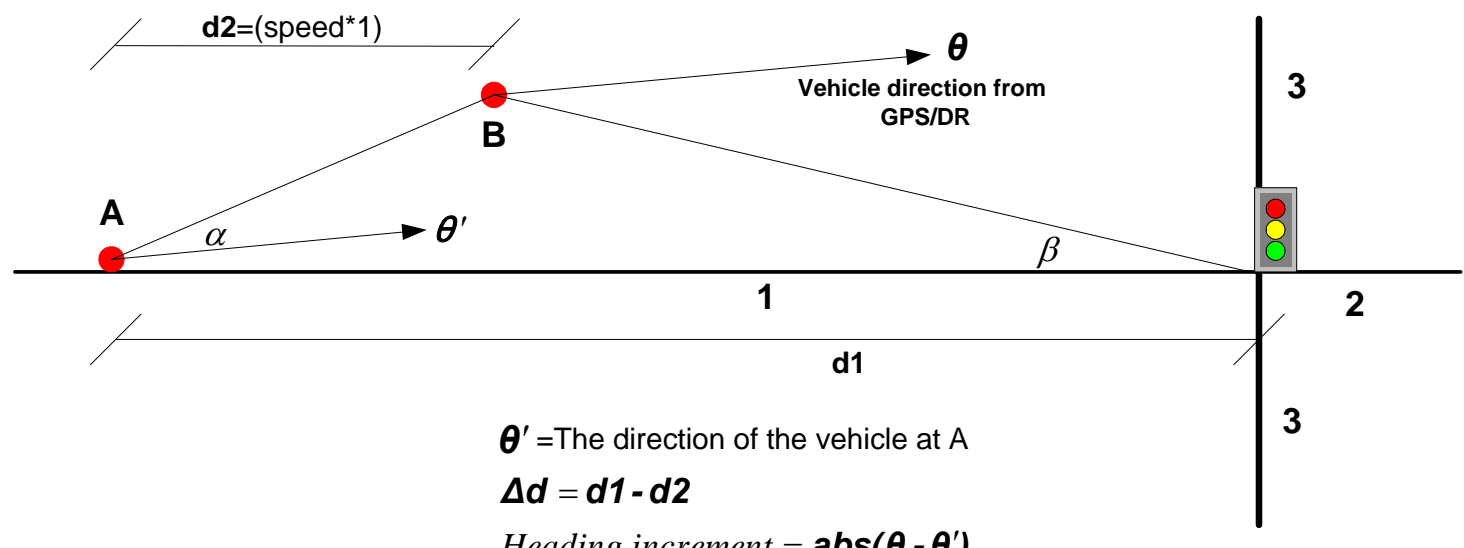

Heading increment $=\boldsymbol{a b s}\left(\boldsymbol{\theta}-\boldsymbol{\theta}^{\prime}\right)$

Figure 5: SMP along a link 


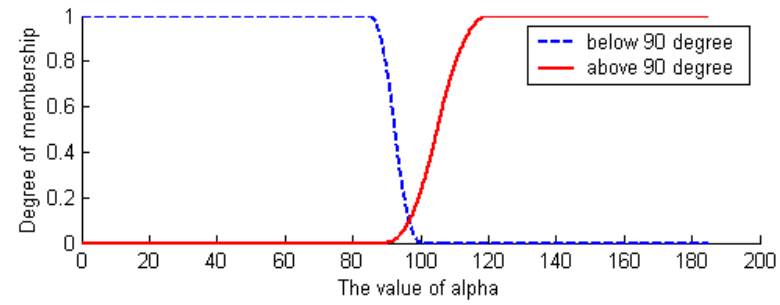

(a)

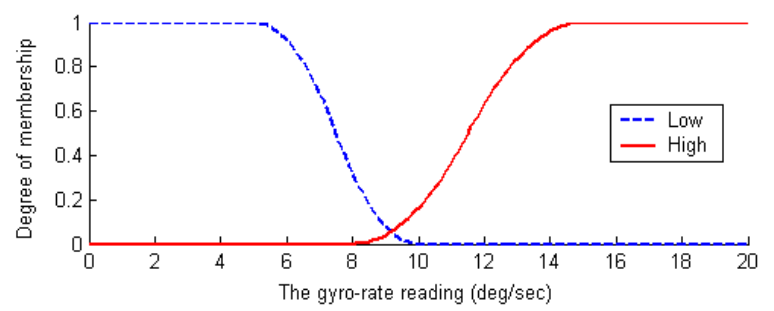

(b)

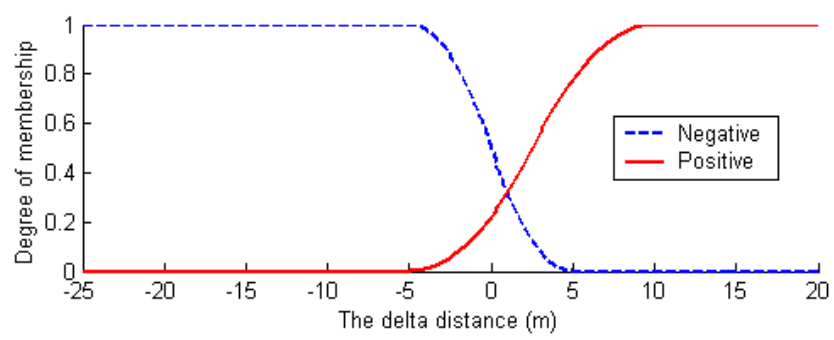

(c) 


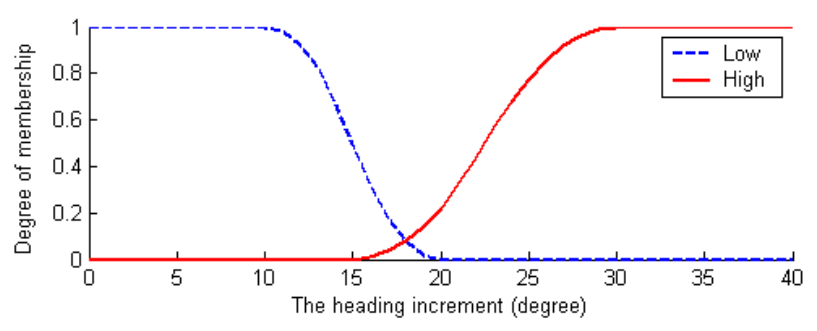

(d)

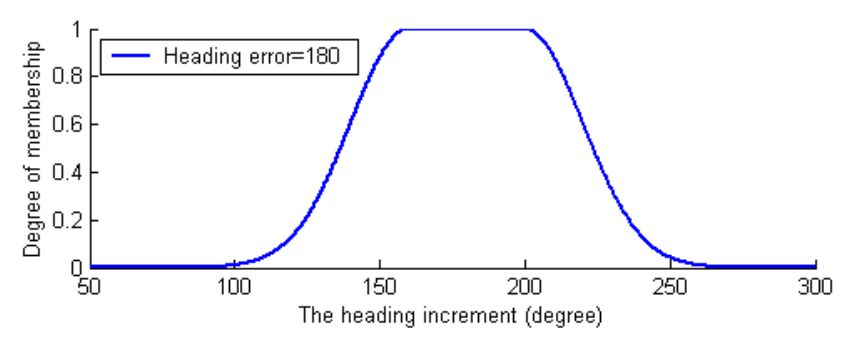

(e)

Figure 6: The MFs for the $\alpha$ or $\beta$ (a), the gyro-rate reading (b) the $\Delta d$ (c), the heading increment (d), and a 180 degree heading increment (e)

$$
\begin{aligned}
& d_{2}=S T+T P \\
& d_{3}=S T+T Q \\
& d_{6}=S T+T U+U R
\end{aligned}
$$

The delta distances :

$$
\begin{aligned}
& \Delta \boldsymbol{d}_{\mathbf{2}}=a b s\left(\boldsymbol{d}-\boldsymbol{d}_{\mathbf{2}}\right) \\
& \boldsymbol{\Delta} \boldsymbol{d}_{\mathbf{3}}=a b s\left(\boldsymbol{d}-\boldsymbol{d}_{\mathbf{3}}\right)
\end{aligned}
$$$$
\Delta \boldsymbol{d}_{6}=a b s\left(\boldsymbol{d}-\boldsymbol{d}_{6}\right)
$$

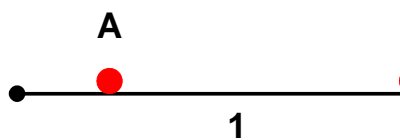

\section{B}

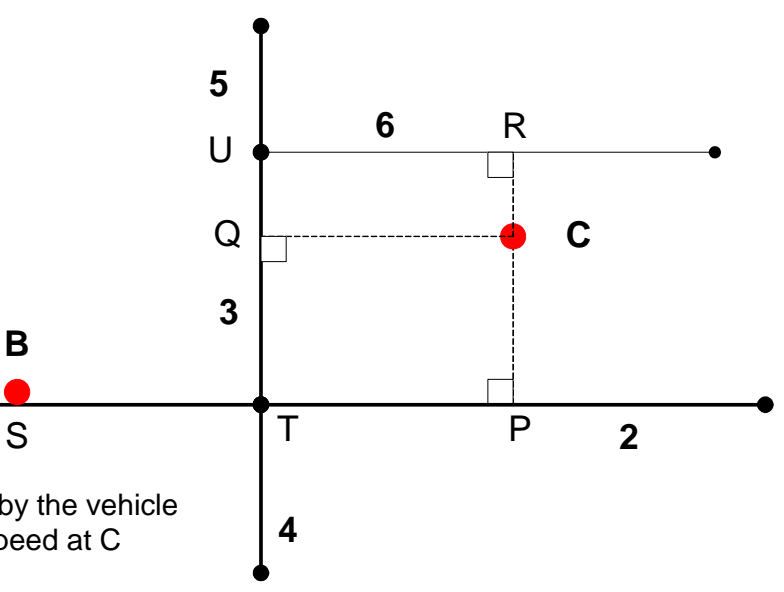

$\boldsymbol{d}=$ The distance traveled by the vehicle calculated from the speed at C

Figure 7: SMP at a junction 


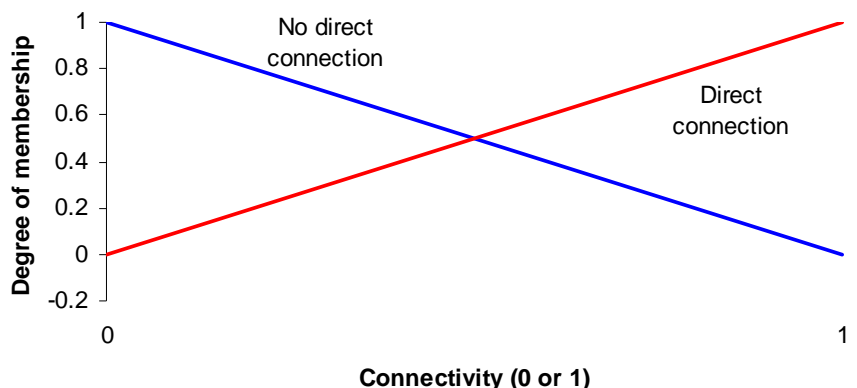


(a)

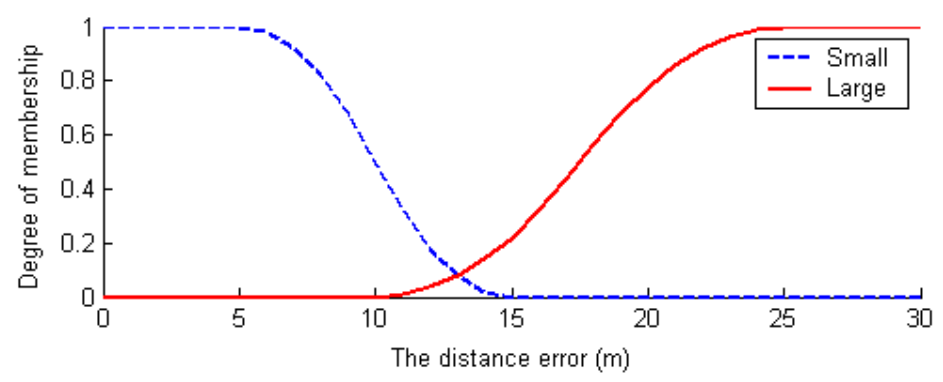

(b)

Figure 8: The MFs for the link connectivity (a), and the delta distance (b) 


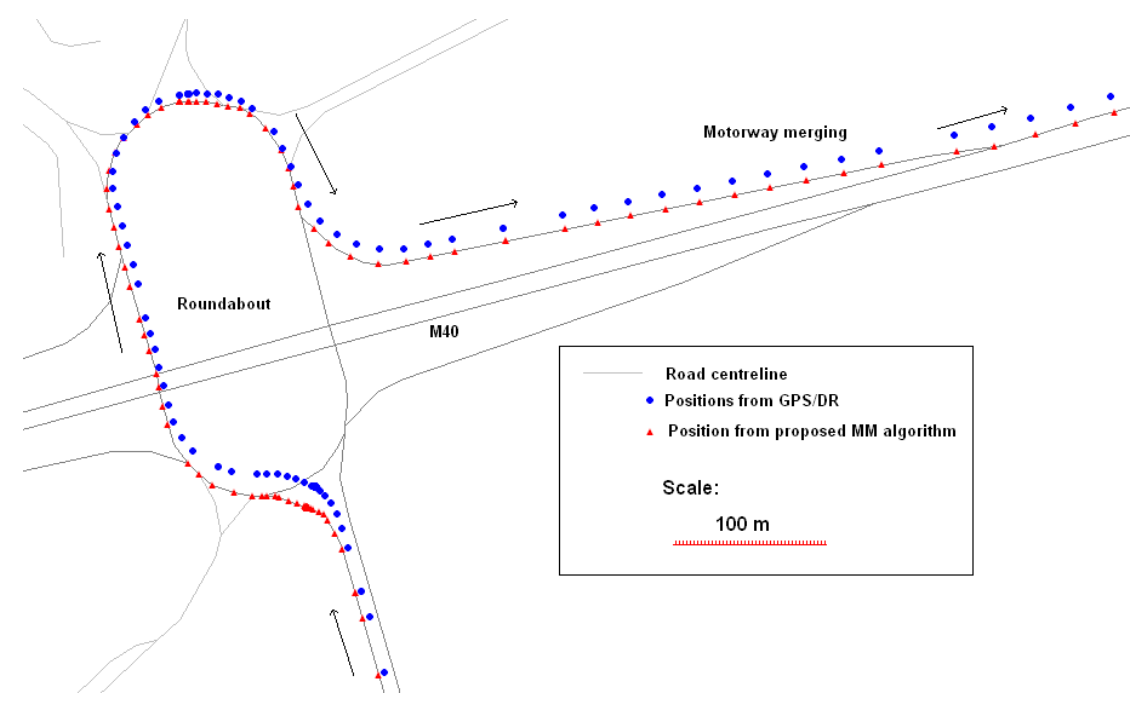

Figure 9: Map matching results for a part of test network which includes a roundabout and a motorway merging 


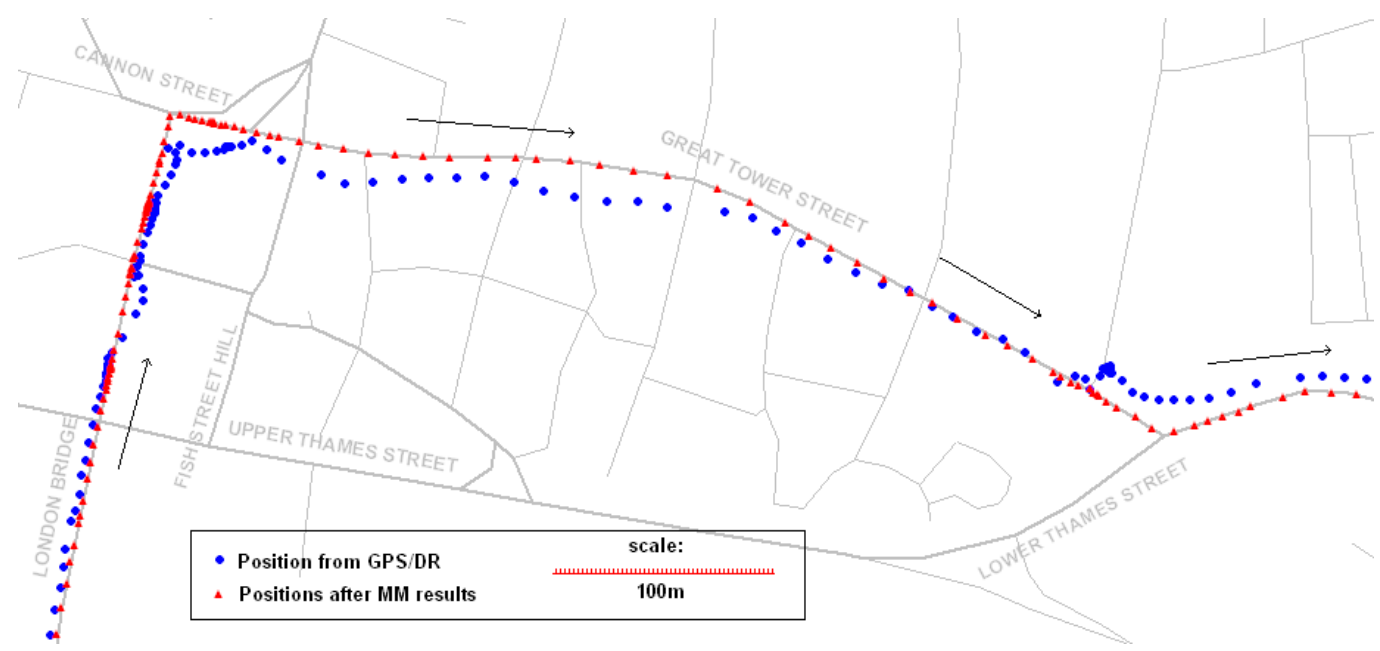

Figure 10: Map matching results for a part of test route in dense urban streets 
Table 1: Performance of map matching algorithms

\begin{tabular}{|l|l|l|l|l|}
\hline Map matching algorithms & Correct link & Horizontal & Along-track & Cross- \\
& Identification & Accuracy & errors \\
& $(\%)$ & $(2 \sigma, m)$ & m) & errors \\
& & & & $(2 \sigma, \mathrm{m})$ \\
\hline Bernstein and Kornhauser (1998) & 70.5 & 46.0 & 45.2 & 10.3 \\
\hline White et al. (2000) & 76.8 & 32.0 & 29.5 & 10.1 \\
\hline Srinivasan et al. (2003) & 80.2 & 21.2 & 18.3 & 10.3 \\
\hline Greenfeld (2002) & 85.6 & 18.3 & 15.5 & 8.6 \\
\hline Yang et al. (2003) & 82.5 & 25.0 & 24.1 & 7.2 \\
\hline Fu et al. (2004) & 80.5 & 23.0 & 22.0 & 8.5 \\
\hline Syed and Cannon (2004) & 92.5 & 16.1 & 15.1 & 5.1 \\
\hline Quddus et al (2003) & 88.6 & 18.1 & 17.6 & 4.8 \\
\hline Ochieng et al. (2004) & 98.1 & 9.1 & 8.2 & 4.0 \\
\hline Fuzzy logic based MM (in this research) & 99.2 & 5.5 & 4.2 & 3.2 \\
\hline
\end{tabular}

